\title{
Controllable Synthesis of $\mathrm{WO}_{3}$ Nanowires by Electrospinning and Their Photocatalytic Properties Under Visible Light Irradiation
}

\author{
Zhe Chen · Wu Wang • Kaigui Zhu
}

Received: 19 December 2013/Revised: 28 April 2014/Published online: 27 September 2014

(C) The Chinese Society for Metals and Springer-Verlag Berlin Heidelberg 2014

\begin{abstract}
Large amounts of $\mathrm{WO}_{3}$ nanowires were prepared on silicon substrates by electrospinning followed by appropriate calcinations in air using ammonium metatungstate (AMT) as $\mathrm{WO}_{3}$ source. Tunable densities and diameters of $\mathrm{WO}_{3}$ nanowires were achieved by changing the electrospinning time and the concentration of AMT in precursor solution. TG/DSC analysis was used to direct the heating process. The effects of both solvent ratio and heating process on the morphology of the obtained nanowires were investigated. The morphology, structure, and chemical compositions of the tungsten oxide were characterized by SEM, XRD, and EDX, respectively. Results showed that monoclinic phase $\mathrm{WO}_{3}$ nanowires with diameters ranging from 100 to $200 \mathrm{~nm}$ were obtained after the appropriate heating process when the AMT concentration of the precursor solution increased from 10 to $20 \mathrm{wt} \%$. The photocatalytic performance of the obtained $\mathrm{WO}_{3}$ nanowires under visible light irradiation $(>420 \mathrm{~nm})$ was investigated in the degradation of Rhodamine B at room temperature in air.
\end{abstract}

KEY WORDS: Nanowires; Electrospinning; Photocatalysis

\section{Introduction}

Due to their distinctive physical, chemical properties, [1-3] and favorable potential applications in various fields [4], one-dimensional materials, such as nanorods, nanobelts, nanofibers, and nanotubes [5-8] have been widely investigated. As one of the transition metal oxides, $\mathrm{WO}_{3}$ is particularly desirable in electrochromic devices, photocatalysts, photoluminescent, field emission and gas sensing materials $[9,10]$. Thermal evaporation [11], templating $[12,13]$, solvothermal [14], and chemical vapor deposition (CVD) [15] have been employed to fabricate $\mathrm{WO}_{3}$ nanowires. However, electrospinning is currently the only

Available online at http://link.springer.com/journal/40195

Z. Chen $\cdot$ W. Wang $\cdot$ K. Zhu $(\bowtie)$

Department of Physics, Beihang University, Beijing 100191, China

e-mail: kgzhu@buaa.edu.cn technique that allows the fabrication of nanoscale continuous fibers [16]. Electrospinning ultrafine fibers with extremely long length and high specific surface area have been extensively applied in many biomedical and industrial fields [16]. Many metal oxide nanomaterials such as $\mathrm{ZnO}$ [17], $\mathrm{SnO}_{2}$, [18] and $\mathrm{TiO}_{2}$ [19] nanowires have been synthesized by electrospinning technology. In addition, oxide semiconductors such as $\mathrm{TiO}_{2}$ have been applied as photocatalysts to stimulate the degradation of environmental toxins due to its high chemical and photoelectrochemical durability [20].

However, $\mathrm{TiO}_{2}$ only can be photoexcited by ultraviolet light that was just (3-5)\% of sunlight due to its large band gap energy (3.2 and $3.0 \mathrm{eV}$ in the anatase and rutile crystalline phases, respectively) [21, 22]. The energy band of $\mathrm{WO}_{3}$ is $2.2-2.8 \mathrm{eV}$ [23-28] and can be photo-excited in the visible region of sunlight, so $\mathrm{WO}_{3}$ is a promising photocatalytic material. The ultra-fine fibers of $\mathrm{WO}_{3}$ were firstly prepared by electrospinning technique based on PVA/ $\mathrm{H}_{3} \mathrm{PWO}_{40}$ gel followed by calcinations at 600 and $800{ }^{\circ} \mathrm{C}$, 
and exhibited excellent photocatalytic activity at $365 \mathrm{~nm}$ wavelength [29]. Different heating processes were used to synthetize $\mathrm{WO}_{3}$ nanowires [29-32]. Proper heating process plays a significant role in the preparation of those materials. Ammonium metatungstate (AMT) was often used as the $\mathrm{WO}_{3}$ source for the preparation of $\mathrm{WO}_{3}$ materials. It is a decomposition of ammonium paratungstate (APT) at 200-250 ${ }^{\circ} \mathrm{C}$ [33]. Therefore, the thermal decomposition of PVA/AMT composites should be similar to that of PVP/ AMT, but it is essential to find appropriate annealing parameters for each polymer/inorganic composite.

In the present study, we report the tunable and convenient synthesis of $\mathrm{WO}_{3}$ nanowires using electrospinning based on PVA/AMT gel followed by calcinations at low temperature $\left(500,550{ }^{\circ} \mathrm{C}\right)$ in air. Thermogravimetric analysis (TG) and differential scanning calorimetry (DSC) were used to study the thermal decomposition process of the precursor solution to optimize the heating process. Scanning electron microscopy (SEM), energy dispersive $\mathrm{X}$-ray spectroscopy (EDX), and X-ray powder diffraction (XRD) were used to investigate the morphology, chemical composition and structure of $\mathrm{WO}_{3}$ nanowires, respectively. Photocatalytic performance of the obtained $\mathrm{WO}_{3}$ nanowires in visible light irradiation was investigated by the photodegradation of Rhodamine $\mathrm{B}(\mathrm{Rh} \mathrm{B})$ in aqueous solution at room temperature.

\section{Experimental}

\subsection{Materials}

Ammonium meta-tungstate hydrate $\left(\left(\mathrm{NH}_{4}\right)_{6} \mathrm{~W}_{7} \mathrm{O}_{24} \cdot 6 \mathrm{H}_{2} \mathrm{O}\right.$, AMT) with a molecular weight of 1887.26 was purchased from the Tianjin City Branch Institute of fine chemicals (Tianjin, China). Poly (vinyl alcohol) $\left(\left[-\mathrm{CH}_{2} \mathrm{CHOH}-\right]_{n}\right.$, PVA) with molecular weight 89,000-98,000 was purchased from Bioszune Life Sciences DEP (US). All the reagents in this experiment were used as received without further treatment.

\subsection{Synthesis of the Ultra-Fine $\mathrm{WO}_{3}$ Nanowires Precursor}

A classical method [34] was used to prepare the AMT/PVA precursor nanofibers. In brief, $0.85 \mathrm{~g}$ PVA was dissolved in $10.625 \mathrm{~g}$ deionized water holding by a $40 \mathrm{~mL}$ beaker with vigorous magnetic stirring for $2 \mathrm{~h}$ at room temperature. Then, 1.063, 1.594, $2.125 \mathrm{~g}$ ammonium metatungstate hydrates were carefully added to the polymer gel $(0.08 \mathrm{~g} /$ $\mathrm{mL}$ ) with another $10 \mathrm{~h}$ vigorous magnetic stirring at room temperature, respectively. The precursor solution with AMT concentration of 10,15 , and $20 \mathrm{wt} \%$ was achieved finally. A $5 \mathrm{~mL}$ plastic syringe with a stainless steel needle (outside diameter $=0.6 \mathrm{~mm}$ ) being grounded using suitable polishing progress was applied to hold the precursor gel. High-voltage power facility was applied to supply the required charge. The anode was connected with the needle tip, and the cathode was linked to a $20 \mathrm{~cm} \times 20 \mathrm{~cm}$ flat aluminum foil. Silicon substrates were used to collect the precursor nanofibres. Acetone, ethanol, deionized water was used to ultrasonically clean the silicon substrates for 5 min, respectively. The distance between the substrates and the needle was maintained at $18 \mathrm{~cm}$. While the gel formed a full-sized droplet with the diameter of about $1 \mu \mathrm{m}$ at the tip of the needle due to its fluidity and gravity, voltage up to $16 \mathrm{kV}$ was applied to the stainless steel needle and the flat aluminum foil.

\subsection{Thermal Properties of the Precursor and Preparation of $\mathrm{WO}_{3}$ Nanowires}

The thermal decomposition of the nanofibers was investigated by a simultaneous thermal analyzer (STA449F3), by which thermal stability, decomposition behavior, proximate analysis, phase transformation, melting process of different materials can be investigated. Heating rate of $10{ }^{\circ} \mathrm{C} / \mathrm{min}$ was used in the range from room temperature to $800{ }^{\circ} \mathrm{C}$ to study the decomposition behavior of the samples.

Based on the TG/DSC results two heating process modes were used. One of the modes (mode A) is: the precursor was calcined at $300{ }^{\circ} \mathrm{C}$ for $1 \mathrm{~h}$, then calcined at $500{ }^{\circ} \mathrm{C}$ for $3 \mathrm{~h}$. The other mode (mode B) is: the precursor was calcined at $300{ }^{\circ} \mathrm{C}$ for $1 \mathrm{~h}$, then calcined at $550{ }^{\circ} \mathrm{C}$ for $3 \mathrm{~h}$. Heating rates between room temperature to 300,500 , and $550{ }^{\circ} \mathrm{C}$ were 2,1 and $1{ }^{\circ} \mathrm{C} / \mathrm{min}$, respectively.

\subsection{Characterization of $\mathrm{WO}_{3}$ Nanowires}

The surface morphology of $\mathrm{WO}_{3}$ nanowires was characterized by SEM (Quanta 250 FEG and JSM-7500F) at an accelerating voltage of $10 \mathrm{kV}$. The composition of chemical elements of $\mathrm{WO}_{3}$ nanowires was measured by energy dispersive EDX. The structure of $\mathrm{WO}_{3}$ nanowires was studied by XRD.

\subsection{Measurement of the Photocatalytic Property of the Obtained $\mathrm{WO}_{3}$ Nanowires}

The photocatalytic property of the obtained nanowires under visible-light was investigated by the degradation of $\mathrm{Rh} \mathrm{B}$ at room temperature in aqueous solution. Typically, $100 \mathrm{mg}$ obtained products and $80 \mathrm{~mL}$ aqueous $\mathrm{Rh} \mathrm{B}$ solution $(0.02 \mathrm{mmol} / \mathrm{L})$ were mixed in a flask and stirred for $1 \mathrm{~h}$ by a magnetic stirrer in order to achieve the 


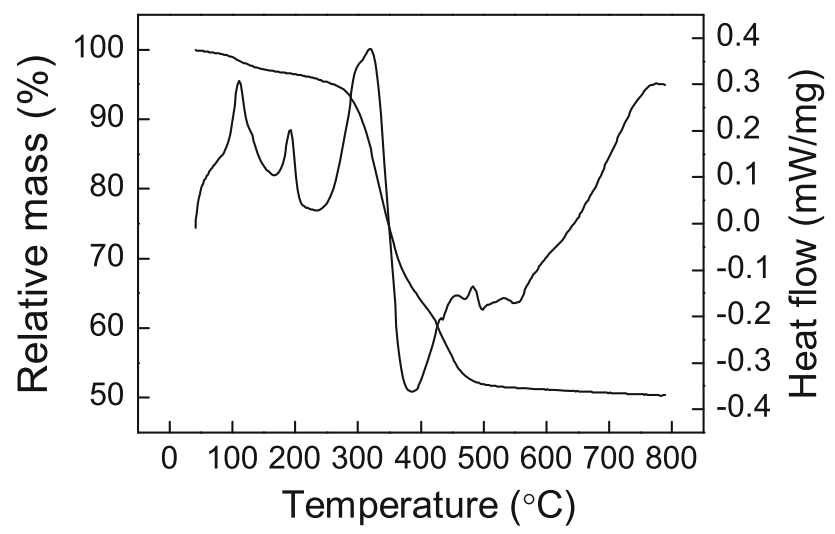

Fig. 1 Simultaneous TG/DSC curves of AMT/PVP nanofibers

adsorption/desorption equilibrium in darkness. Then the nanowire suspension solution was irradiated for $3.5 \mathrm{~h}$ using a Xenon lamp $(350 \mathrm{~W})$ under continuous stirring. A light filter was used to eliminate light wave below $420 \mathrm{~nm}$. About $3 \mathrm{~mL}$ suspension solution was retrieved using a disposable pipette every half hour, and analyzed by a double beam UV-Vis spectrophotometer to determine the absorption intensity of $\mathrm{Rh} \mathrm{B}$ at the wavelength of $554 \mathrm{~nm}$ after necessary separation of the nanowires using a centrifugal machine.

\section{Results and Discussion}

\section{$3.1 \mathrm{TG} / \mathrm{DSC}$}

The TG/DSC curves of the thermal decomposition of AMT/PVP nanofibres are shown in Fig. 1. When the annealing temperature increased to $500{ }^{\circ} \mathrm{C}$, the weight of composite fibers was about $50 \%$ of the original weight and did not decrease furthermore indicating that the PVA/AMT compound was decomposed completely. The adsorbed water was released from both PVA and AMT (5\% mass loss) between 40 and $\sim 275{ }^{\circ} \mathrm{C}$. The largest mass loss occurred (about 50\%) between 275 and $500{ }^{\circ} \mathrm{C}$ due to the decomposition of PVA and AMT. Then there was no further mass loss was observed at higher temperature up to $800{ }^{\circ} \mathrm{C}$. The result showed that there were violent chemical reactions between 275 and $500{ }^{\circ} \mathrm{C}$. So, in order to obtain excellent morphology of $\mathrm{WO}_{3}$ nanowires, holding $1 \mathrm{~h}$ at $300{ }^{\circ} \mathrm{C}$ and lower heating rate $\left(1{ }^{\circ} \mathrm{C} / \mathrm{min}\right)$ above $300{ }^{\circ} \mathrm{C}$ were used in the present study. However, there was an endothermic peak at $\sim 750{ }^{\circ} \mathrm{C}$ in the DSC curve. That may due to the phase transition. According to previous research, crystalline $\mathrm{WO}_{3}$ is a tetragonal above $740{ }^{\circ} \mathrm{C}$ [35]. Phase transition significantly controls the photocatalytic activities, such as in $\mathrm{CuO}-\mathrm{K}_{2} \mathrm{Mo}_{4} \mathrm{O}_{13}$ systems [36]. However, there was no significant influence in the present study. So we have not further investigated that in detail.

\subsection{SEM and EDX}

Due to the fluidity and the gravity of the precursor solution, the gel formed a full-sized droplet at the tip of the needle before the electrospinning process. When the high voltage turned on, the accumulation of electrostatic charges on the surface of the droplet stretched the droplet to be a continuous fiber. During the proper annealing process the obtained fibers were transformed to $\mathrm{WO}_{3}$ nanowires.

Figure 2 showed SEM imagines of the nanowires calcined for $3 \mathrm{~h}$ at $500{ }^{\circ} \mathrm{C}$ (Fig. 2a, b) and $550{ }^{\circ} \mathrm{C}$ (Fig. 2c-f). The concentrations of AMT in the precursor solution of samples a, b, c, d, e, and f were 10, 15, 10, 15, 20, and $20 \mathrm{wt} \%$, respectively. Figure 2 indicates that the diameter of the products increases with the concentration of AMT increasing. The diameter varied from 100 to $\sim 200 \mathrm{~nm}$ when the concentration of AMT increased from 10 to $20 \mathrm{wt} \%$. The average diameter of fibrous $\mathrm{WO}_{3}$ was varied from 200 to $600 \mathrm{~nm}$ after calcined $\mathrm{PVA} / \mathrm{H}_{3} \mathrm{PW}_{12} \mathrm{O}_{40}$ fibers at 600 and $800{ }^{\circ} \mathrm{C}$, respectively [29]. The diameter of the products was smaller comparing to [29] and the morphology of the nanowires was smooth. However the morphology of samples shown in Fig. 2a, b were smoother than samples shown in Fig. 2a, d, e. What's more, the grains of the latter were more distinct than the former. That indicated that a segregation process caused by a heating process from 500 to $550{ }^{\circ} \mathrm{C}$. It should be pointed out that the heating process unlikely broke the nanowires although slight agglomeration happened. The agglomeration is a popular phenomenon in the heating progress of samples preparation, and mesoporous tungsten oxide nanofibers [30] were prepared based on that behavior. Apparently, the nanowires calcined at higher temperature have the risk of fracture.

The EDX spectrum of the nanowires calcined for $3 \mathrm{~h}$ at $550{ }^{\circ} \mathrm{C}$ were also measured, as shown in Fig. 3. The results confirmed that tungsten was the only element except oxygen and silicon in the sample after calcinations indicated PVA/AMT compounds have decomposed completely. The signal of silicon was risen from the silicon substrates.

\subsection{XRD}

Figure 4 showed the XRD spectrum of the calcined PVA/ AMT compounds at $550{ }^{\circ} \mathrm{C}$ for $3 \mathrm{~h}$. It showed that the products were only consist of monoclinic phase $\mathrm{WO}_{3}$ $(7.297 \times 7.539 \times 7.688\langle 90.91\rangle$, JCPDS 43-1035) [37]. No evidence of other crystalline phases was shown in the XRD pattern indicating that the AMT/PVA compounds were decomposed completely, which was coincident with the EDX analysis. 

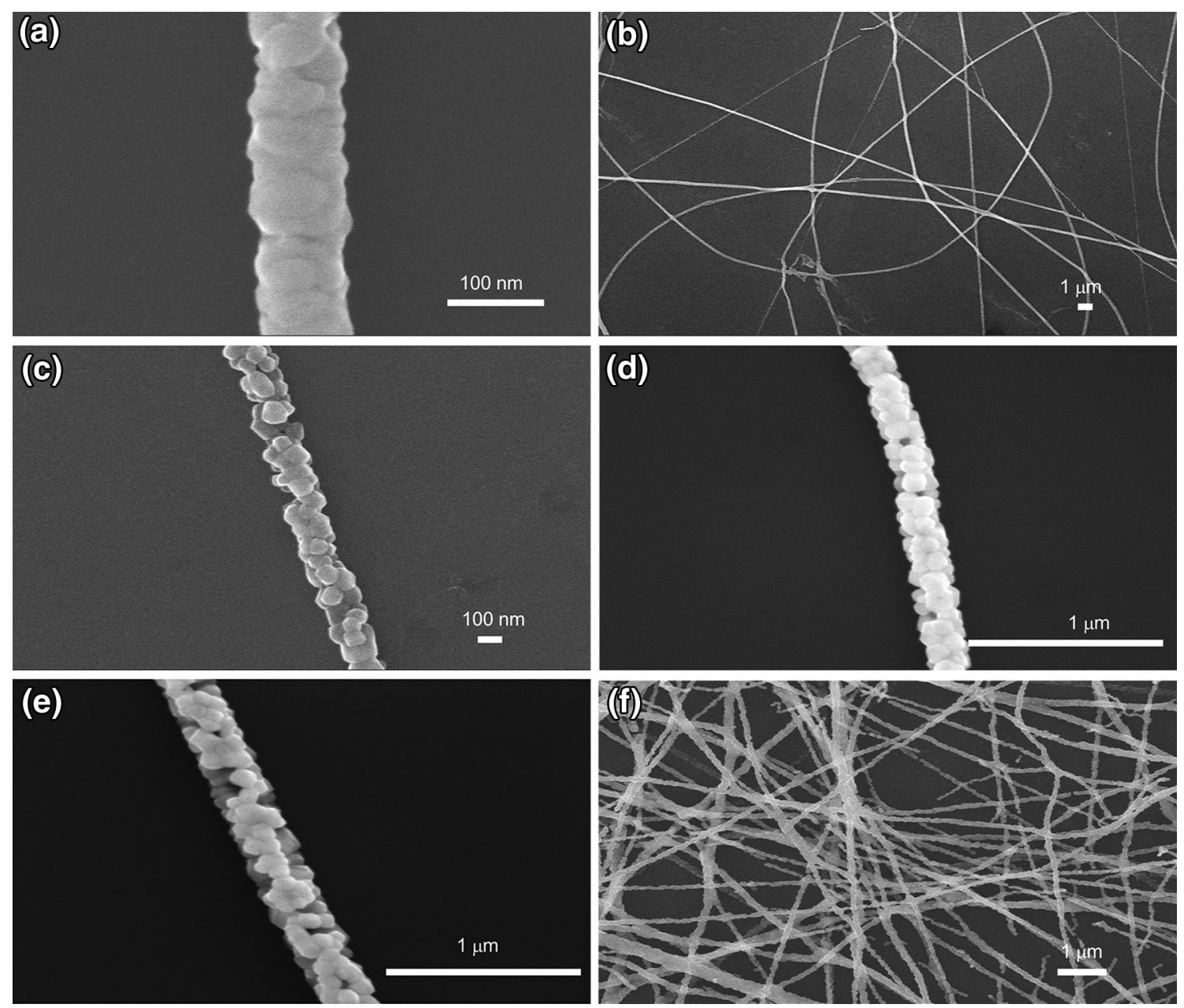

Fig. 2 SEM imagines of the nanowires calcined in mode A a, b and in mode B c-f

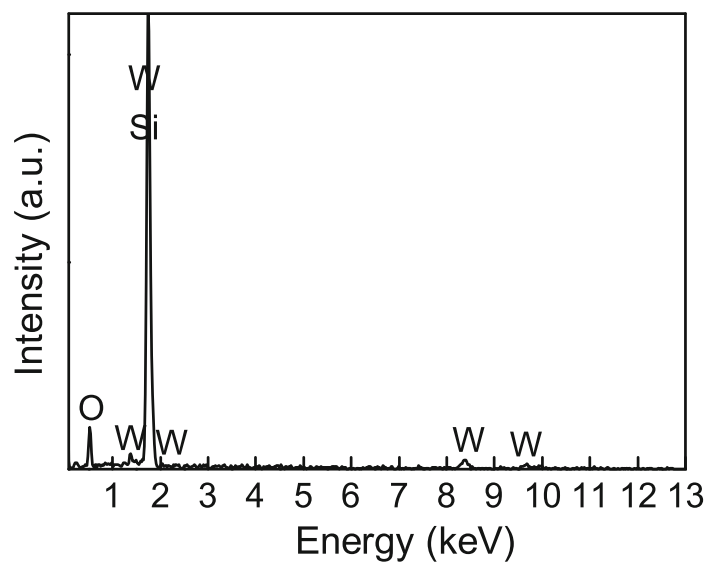

Fig. 3 EDX spectrum of the nanowires calcined for $3 \mathrm{~h}$ at mode B

\subsection{Photocatalytic Activity}

Figure 5 showed the photocatalytic degradation rate of $\mathrm{Rh}$ $\mathrm{B}$ at room temperature in aqueous solution. The absorption

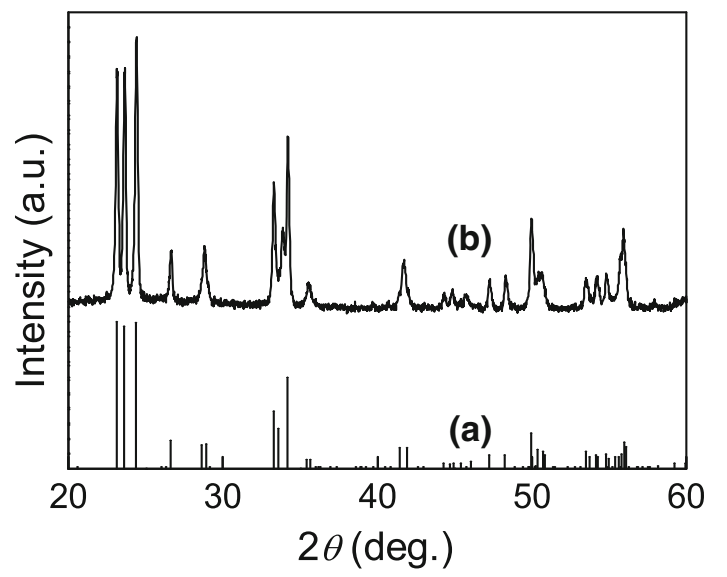

Fig. 4 XRD curves of monoclinic $\mathrm{WO}_{3}$ a and products calcined at mode $\mathrm{B} \mathbf{b}$

intensity of Rh B was only slightly decreased after the adsorption/desorption equilibrium and have no remarkable changes in the absence of photocatalytic samples under 


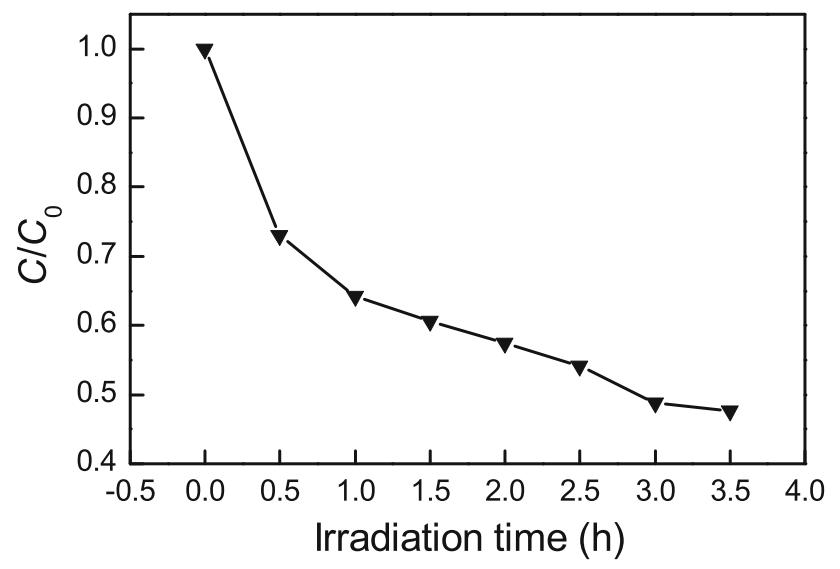

Fig. 5 Photocatalytic degradation rate of $\mathrm{Rh} \mathrm{B}$ under the light $(>420 \mathrm{~nm})$ irradiation

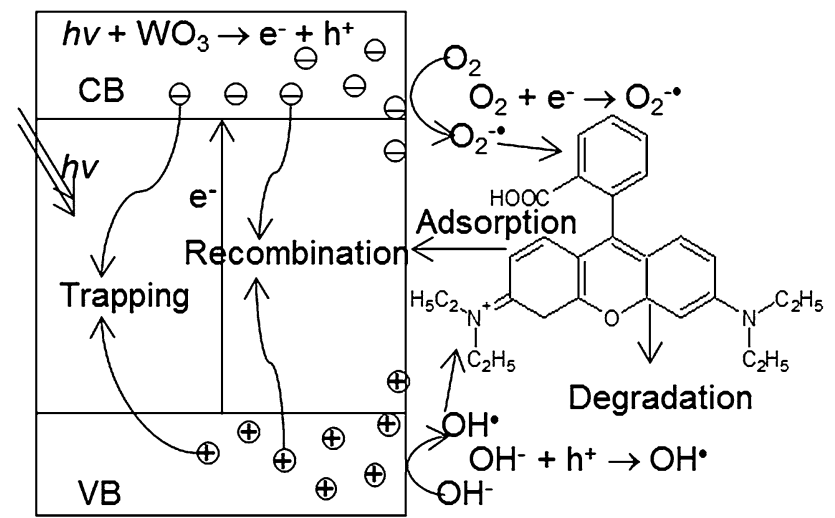

Fig. 6 The degradation mechanism of Rh B under light irradiation

dark conditions [21]. So the sharp degradation of $\mathrm{Rh} \mathrm{B}$ is due to the catalysis of $\mathrm{WO}_{3}$ nanowires. The concentration of $\mathrm{Rh} \mathrm{B}$ dye was degraded about $48 \%$ by the $\mathrm{WO}_{3}$ nanowires after $3.5 \mathrm{~h}$ irradiation at Xenon lamp $(350 \mathrm{~W})$ with a light filter to eliminate the wavelength below $420 \mathrm{~nm}$. Researches usually did irradiation experiments of $\mathrm{WO}_{3}$ nanowires with UV light (365 nm) [23, 29]. However, the photocatalytic property of the samples under visible light was studied in the present work. The photodegradation of $\mathrm{Rh} \mathrm{B}$ cannot reach to $50 \%$ with $4 \mathrm{~h}$ irradiation under a $250 \mathrm{~W}$ high-pressure mercury lamp [38]. The photodegradation of $\mathrm{Rh} \mathrm{B}$ reaches to $50 \%$ under the irradiation of a $500 \mathrm{~W}$ Xenon lamp with $2.0 \mathrm{~g} / \mathrm{L} \mathrm{MFO} / \mathrm{TiO}_{2}$ composites calcined at 473 and $873 \mathrm{~K}$ [39]. The excellent photocatalytic performance in degradation of $\mathrm{Rh} \mathrm{B}$ in aqueous solution under light irradiation above $420 \mathrm{~nm}$ wavelength could be due to the small parameter (100-200 nm). When $h v \geq\left(E_{\mathrm{C}}-E_{\mathrm{V}}\right)$, where $E_{\mathrm{C}}$ and $E_{\mathrm{V}}$ are the energies for the conduction band bottom (VB) and the valence band (CB) top of $\mathrm{WO}_{3}$ nanowires respectively, electrons can be excited into the valence band of $\mathrm{WO}_{3}$ while producing holes. Then they can migrate to the surface of the nanowires and react with the adsorbed $\mathrm{O}_{2}$ and $\mathrm{H}_{2} \mathrm{O}$ forming $\mathrm{O}_{2}^{-}$and $\mathrm{OH}^{\bullet}$ to degrade $\mathrm{Rh} \mathrm{B}$. The detailed mechanisms of the degradation of Rh B are shown in Fig. 6. The photocatalytic property is determined by the completion of trapping, recombination and transfer capability to surface of electron-hole pairs [40]. The diameters of the nanowires were only $100-200 \mathrm{~nm}$, the recombination of electrons and holes can be reduced compared to large size or bulk. Therefore, more electrons and holes can transfer to surface to yield radicals, and the nanowires have good adsorbability of $\mathrm{O}_{2}$ and $\mathrm{H}_{2} \mathrm{O}$ due to large surface area. In addition, the light reflectivity of nanowire is very low due to the small-size effect. Therefore, more light can be trapped to trigger the degradation of $\mathrm{Rh} \mathrm{B}$. That indicated that the light above $420 \mathrm{~nm}$ was sufficient to excite electrons in the valence band of the obtained $\mathrm{WO}_{3}$ nanowires. However the role for $\mathrm{WO}_{3}$ nanowires on the formation of $\mathrm{O}_{2}^{-}$and $\mathrm{OH}^{\bullet}$ to degrade $\mathrm{Rh} \mathrm{B}$ still needs more investigation.

\section{Conclusions}

Large amounts of $\mathrm{WO}_{3}$ nanowires are easily prepared by electrospinning followed by appropriate heating processes $\left(500,550{ }^{\circ} \mathrm{C}\right)$ in air on silicon substrates using AMT/PVA precursors. The products were characterized by XRD, SEM, and EDX. The heating process was optimized according to the TG/DSC results. Monoclinic phase $\mathrm{WO}_{3}$ nanowires with varied diameters from 100 to $200 \mathrm{~nm}$ were obtained after the appropriate heating process when the concentration of AMT increased from 10 to $20 \mathrm{wt} \%$. The surface of samples was smooth and the small grains of nanowires did not show separation after appropriate calcinations. The high degradation rate of $\mathrm{Rh} \mathrm{B}$ was obtained in aqueous solution under visible light $(>420 \mathrm{~nm})$ irradiation due to the small size samples catalysis. $\mathrm{WO}_{3}$ nanowires fabricated by electrospinning have the potential to be a promising photocatalytic material due to the simple synthetic process, controllable parameters and excellent photocatalytic performance.

Acknowledgments This work was financially supported by the project from the National Magnetic Confinement Fusion Program (No. 2011GB108008) and the Natural Science Foundation of China (No. 51171006).

\section{References}

[1] Y. Xia, P. Yang, Y. Sun, Y. Wu, B. Mayers, B. Gates, Y. Yin, F. Kim, H. Yan, Adv. Mater. 15, 353 (2003)

[2] A.P. Alivisatos, Science 271, 933 (1996) 
[3] J. Zhou, Y. Ding, S.Z. Deng, L. Gong, N.S. Xu, Adv. Mater. 17, 2107 (2005)

[4] X. Duan, Y. Huang, Y. Cui, J. Wang, C.M. Lieber, Nature 409, $66(2001)$

[5] H.J. Dai, E.W. Wong, Y.Z. Lu, S.S. Fan, C.M. Lieber, Nature 375, 769 (1995)

[6] Y. Su, B. Lu, Nanotechnology 22, 285609 (2011)

[7] G.S. Bisht, G. Canton, A. Mirsepassi, L. Kulinsky, S. Oh, D. Dunn-Rankin, M.J. Madou, Nano Lett. 11, 1831 (2011)

[8] H.B. Peng, T.G. Ristroph, G.M. Schurmann, G.M. King, J. Yoon, V. Narayanamurti, J.A. Golovchenko, Appl. Phys. Lett. 83, 4238 (2003)

[9] K.J. Lethy, D. Beena, R. Vinod, Kumar, V.P. Mahadevan Pillai, V. Ganesan, V. Sathe, Appl. Surf. Sci. 254, 2369 (2008)

[10] Y.S. Kim, S.L. Ha, K. Kim, H. Yang, S.-Y. Choi, Y.T. Kim, J.T. Park, C.H. Lee, J. Choi, J. Paek, k. Lee, Appl. Phys. Lett. 86, 213105 (2005)

[11] A. Ponzoni, E. Comini, G. Sberveglieri, J. Zhou, S.Z. Deng, N.S. Xu, Y. Ding, Z.L. Wang, Appl. Phys. Lett. 88, 203101 (2006)

[12] K. Zhu, H. He, S. Xie, X. Zhang, W. Zhou, S. Jin, B. Yue, Chem. Phys. Lett. 377, 317 (2003)

[13] Y. Qin, F. Wang, W. Shen, M. Hu, J. Alloys Compd. 540, 21 (2012)

[14] H.G. Choi, Y.H. Jung, D.K. Kim, J. Am. Ceram. Soc. 88, 1684 (2005)

[15] X.L. Li, J.F. Liu, Y.D. Li, Inorg. Chem. 42, 921 (2003)

[16] D. Zhang, J. Chang, Nano Lett. 8, 3283 (2008)

[17] D. Lin, H. Wu, W. Pan, Adv. Mater. 19, 3968 (2007)

[18] L. Li, X. Yin, S. Liu, Electrochem. Commun. 12, 1383 (2010)

[19] L.E. Greene, M. Law, B.D. Yuhas, P. Yang, J. Am. Ceram. Soc. 111, 18451 (2007)

[20] D. Vernardou, H. Drosos, E. Spanakis, E. Koudoumas, C. Savvakis, N. Katsarakis, J. Mater. Chem. 21, 513 (2011)

[21] Y. Li, X. Zhou, W. Chen, L. Li, M. Zen, S. Qin, S. Sun, J. Hazard. Mater. 227-228, 25 (2012)
[22] Y. Meng, J. Chen, Y. Wang, H. Dingy, Y. Shan, J. Mater. Sci. Technol. 25, 73 (2009)

[23] M. Qamar, M.A. Gondal, K. Hayat, Z.H. Yamani, K. AlHooshani, J. Hazard. Mater. 170, 584 (2009)

[24] X. Gang, G. Wei, T. Ma, Appl. Surf. Sci. 256, 165 (2009)

[25] S. Wang, X. Shi, G. Shao, X. Duan, H. Yang, T. Wang, J. Phys. Chem. Solids 69, 2396 (2008)

[26] H.Y. Wang, P. Xu, T.M. Wang, Mater. Des. 23, 331 (2002)

[27] M.A. Gondal, A. Dastageer, A. Khalil, Catal. Commun. 11, 214 (2009)

[28] M.A. Gondal, M.N. Sayeed, A. Arfaj, Chem. Phys. Lett. 445, 325 (2007)

[29] C. Sui, J. Gong, T. Cheng, G. Zhou, S. Dong, Appl. Surf. Sci. 257, 8600 (2011)

[30] T.-A. Nguyen, T.-S. Jun, M. Rashid, Y.S. Kim, Mater. Lett. 65, $2823(2011)$

[31] G. Wang, Y. Ji, X. Huang, X. Yang, P.I. Gouma, M. Dudley, J. Phys. Chem. B 110, 23777 (2006)

[32] J. Leng, X. Xu, N. Lv, H. Fan, T. Zhang, J. Colloid Interface Sci. 356, 54 (2011)

[33] S.A.A. Mansour., M.A. Mohamed, M.I. Zaki, Thermochim. Acta 129, 187 (1988)

[34] W.E. Teo, S. Ramakrishna, Nanotechnology 16, 1878 (2005)

[35] M. Boulova, G. Lucazeau, J. Solid State. Chem. 167, 425 (2002)

[36] M. Kassem, Acta Metall. Sin. (Engl. Lett.) 27, 180 (2014)

[37] I.M. Szilagyi, J. Madarasz, G. Pokol, P. Kiraly, G. Tarkanyi, S. Saukko, J. Mizsei, A.L. Toth, A. Szabo, K. Varga-Josepovitso, Chem. Mater. 20, 4116 (2008)

[38] Q.F. Meng, K.R. Liu, L. Jiang, Q. Han, J.S. Chen, X.J. Wei, Acta Metall. Sin. (Engl. Lett.) 17, 263 (2004)

[39] L. Zhang, Y. He, Y. Wu, T. Wu, Mater. Sci. Eng. B 176, 1497 (2011)

[40] J. Zhuang, W. Dai, Q. Tian, Z. Li, L. Xie, J. Wang, P. Liu, Langmuir 26, 9686 (2010) 